\title{
Atividade in vitro de anti-helmínticos sobre larvas infectantes de nematódeos gastrintestinais de caprinos, utilizando a técnica de coprocultura quantitativa (Ueno, 1995)
}

\author{
CÁSSIA CRISTINA LEAL BORGES*
}

\begin{abstract}
ANTHELMINTIC'S ACTIVITIES IN VITRO ABOUT INFECTED WORMS FROM CAPRINE GASTROINTESTINAL NEMATODES USING QUANTITATIVE FAECES CULTURE TECHNIC
\end{abstract}

(UENO, 1995)

This work was achieve with the objective of the compare the action ovi-larvicida in vitro of anthelmintic, using the caprine gastrointestinal nematodes quantitative faeces culture technic. Seven anthelmintics were testes, in the experiment: albendazole, albendazole sulfoxide, fenbendazole, oxfendazole, ivermectin, levamisole and closantel. The used goats for obtain of the faeces, had been kept inthe school of the Medicina Veterinary/University Federal of Bahia(UFBA), for one year and middle, without receiving anthelmintics. The anthelmintics.were dilutes in destiled water, from the original solution, initiating itsell of the dilution 1:10 until 1:5120, perform in the total of the 10 dilutions. The faecal culture prepared with $2 \mathrm{~g}$ of the faeces, $2 \mathrm{~g}$ of the sawing and $2 \mathrm{ml}$ of the distil water ar the differents dilutions of the anthelmintics, had been maintained the $34^{\circ} \mathrm{C}$, for seven days, for obtain of the infective larvae of the gastrointestinal nematodes. The ivermectin presented ovilarvicida action better, with reduction genus of the larvae Haemonchus, Trichostrongylus and Oesophagostomum $\left(L_{3}\right)$, white the fenbendazole was the product with action smaller for the genus Haemonchus and Trichostrongylus. For the genus Oesophagostomum, the products albendazole, fenbendazole, oxfendazole and albendazole sulfoxide, had full eliminated the population of this species. The products between ivermectin, levamisole and closantel, the ivermectin presented action better ovi-larvicida for eggs of the Oesophagostomum.

Key words: Antihelmintics. Evaluation "in vitro", Ovi-larvicida effect. Goats.

\section{INTRODUÇÃO}

A cabra foi o primeiro doméstico capaz de produzir alimentos, há cerca de dez mil anos, acompanhando sempre a história da humanidade, conforme atestam os diversos relatos históricos, mitológicos e até mesmo bíblicos.

A região semi-árida do Estado da Bahia é caracterizada por possuir o maior rebanho caprino nacional, onde a caprinocultura é predominan-

Zootecnista

E-mail: cassiabor@zipmail.com.br 
temente de corte, caracterizada pelo sistema de produção extensiva e às vezes ultra-extensiva. $\mathrm{O}$ rebanho caprino do Estado da Bahia ocupa o primeiro lugar no Brasil (39,92\%); e representa $54,03 \%$ da população caprina do Nordeste Brasileiro ${ }^{1}$.

Vários princípios ativos de anti-helmínticos vêm sendo utilizados no tratamento de nematodeoses, principalmente os grupos dos benzimidazóis (Albendazole, Fenbendazole e Oxfendazole); das avermectinas (Ivermectina); dos imidazotiazoles (Cloridrato de levamisole) e das salicilanilideos (Closantel sódico).

Os prejuízos à caprinocultura nacional causados pelos nematódeos gastrintestinais são mais evidentes na região Nordeste, onde a exploração desta espécie animal é mais intensa e de relevante importância social. A utilização de anti-helmíntico contra nematódeos gastrintestinais de pequenos ruminantes, especialmente caprinos, é indispensável nas regiões tropicais úmidas, levando a maioria dos criadores a aplicarem diversos grupos de anti-helmínticos com várias dosificações por ano, e que inevitavelmente, pode causar diminuição da eficácia do produto ${ }^{2}$.

\section{MATERIAL E MÉTODOS}

$\mathrm{O}$ experimento foi realizado na Escola de Medicina Veterinária da Universidade Federal da Bahia (UFBA) no Laboratório de Diagnóstico das Parasitoses dos Animais, entre dezembro de 1996 e junho de 1997.

Foi realizado atividade in vitro de benzimidazois, imidazotiazole, tetraidropirimidinas e avermectinas sobre larvas infectantes de nematódeos gastrintestinais utilizando a técnica de coprocultura quantitativa, onde foram utilizados os seguintes produtos químicos: albendazole ${ }^{1}-10 \mathrm{~g} / 100 \mathrm{~mL}$, sulfoxido de albendazole $^{2}-6 \mathrm{~g} / 100 \mathrm{~mL}$, fenbendazole $\mathrm{e}^{3}-3,3 \mathrm{~g} /$ $100 \mathrm{~mL}$, oxfendazole ${ }^{4}-2,265 \mathrm{~g} / 100 \mathrm{~mL}$, ivermectin $^{5}-800 \mathrm{mcg} / \mathrm{mL}$, levamisole ${ }^{6}-5 \mathrm{~g} / 100$ $\mathrm{mL}$ e closantel ${ }^{7}-10 \mathrm{~g} / 100 \mathrm{~mL}$.
Foram utilizados quatro caprinos, Pardo Alpino, com idade aproximadamente de 6 a 7 meses, infectados naturalmente para obtenção das fezes, sendo dois para cada experimento. Os animais foram criados semi-intensivamente na Escola de Medicina Veterinária/UFBA, sem dosificações anti-helmínticas. As amostras de fezes foram coletadas individualmente, diretamente da ampola retal dos animais de aproximadamente $30 \mathrm{~g}$, em coletas sucessivas.

A partir da concentração comercial dos antihelmínticos, foram efetuadas diluições em água destilada $1: 10 ; 1: 20 ; 1: 40 ; 1: 80 ; 1: 160 ; 1: 320$; $1: 640 ; 1: 1280 ; 1: 2560 ; 1: 5120$. Para cada diluição foram utilizados dois cultivos de larvas, perfazendo um total de 20 cultivos por produto. Os dois cultivos para o grupo controle foi tratado com água destilada.

Os exames coprológicos foram realizados imediatamente após coleta das amostras fecais. Para a contagem de ovos por grama de fezes (opg) de nematódeos gastrintestinais, foi empregada a técnica ${ }^{3}$ modificada $^{4}$, utilizando-se $2 \mathrm{~g}$ de fezes de caprinos diluídas em $58 \mathrm{ml}$ de solução hipersaturada de açúcar. $\mathrm{O}$ cultivo de larvas de nematódeos gastrintestinais foi realizado pelo método de coprocultura quantitativa descrita por Ueno (1995), que consistia em pesar $2 \mathrm{~g}$ de fezes e adicionar $2 \mathrm{~mL}$ do produto químico nos cultivos tratados ou $2 \mathrm{~mL}$ de água destilada nos cultivos controles. Os grupos de cultivo por produtos, foram armazenados, por uma hora, em caixa plástica (20 x $14 \times 8 \mathrm{~cm}$ de altura) cuja tampa apresentava orifícios na sua superfície, e o seu interior coberto com uma toalha de papel umidecida com água, para conservar a umidade, e mantidos no ambiente do laboratório. Após este período, em cada amostra foi adicionada $2 \mathrm{~g}$ de serragem lavada e esterilizada, homogeinizada e transferida para o copo de vidro $(6 \mathrm{~cm}$ de altura $\mathrm{x} 4,5 \mathrm{~cm}$ de diâmetro) que foi coberto com um filme de PVC, sendo efetuados orifícios pequenos no filme para aeração dos cultivos. Os cultivos foram novamente armazenados nas caixas de

Endazol®- IRFA (Química e Biotecnologia Industrial Ltda)

Ricobendazole ${ }^{\circledR}$ - Produtos Veterinários Ouro Fino Ltda.

Panacur®- Hoechst do Brasil Química e Farmacêutica AS.

Systamex ${ }^{\circledR}-$ Mallinckrodt Veterinary Ltda

Ivomec®- Merck Sharp e Dohme Farmacêutica e Veterinária Ltda

Ripercol®- Cyanamid

Diantel®- IRFA (Química e Biotecnologia Industrial Ltda) 
plástico e permaneceram em estufa à $34^{\circ} \mathrm{C}$ durante sete dias. No 4을 dia de cultivo larval, foi procedida nova umidificação da toalha de papel no interior da caixa.

No sétimo dia da realização da coprocultura as larvas infectantes de nematódeos gastrintestinais foram coletadas em um tubo de ensaio e permaneceram sob refrigeração (aproximadamente $8^{\circ} \mathrm{C}$ ) até o momento da identificação, seguindo descrição ${ }^{6}$ e a contagem que for efetuando, utilizando-se em lâmina de vidro milimetrada, após inativação das larvas com lugol. Nas amostras em que a quantidade de larvas era reduzida a contagem foi efetuada em sua totalidade. Quando obteve um grande número de larvas, o volume do cultivo no tubo de ensaio foi completado com água para $5 \mathrm{ml}$, e homogeneizado e colhido uma amostra de 0,5 $\mathrm{ml}$ para contagem das larvas. $\mathrm{O}$ número de larvas encontradas foi multiplicado por 10. O número total das larvas observadas foi então dividido por dois, e o resultado expresso em larvas por grama de fezes (LPG).

A fórmula abaixo descrita foi empregada para se determinar a percentagem de redução do número de larvas por grama de fezes.

\section{$\%$ Eficácia $=\underline{\text { LDPG do grupo tratado }} \times 100$ LDPG do grupo controle}

Para análise estatística o número total de larvas infectantes de nematódeos gastrintestinais, foi transformado por meio da equação $\mathrm{Y}=\log (\mathrm{y}+1)$.

Foi utilizado o procedimento do Modelo linear (GLM) do programa estatístico (SAS), testandose os efeitos dos caprinos, considerando como blocos, e dos fatores produto e diluição bem como sua interação. Para comparação entre médias foi utilizado o procedimento Média de Míninos Quadrados (LSMEANS). O fator diluição foi considerado também como variável regressora, ajustando-se a curva para cada produto.

\section{RESULTADOS}

Conforme Tabela 1 , o produto $\mathrm{ABZ}^{2}$ mostrou melhor ação ovi-larvicida entre os produtos a base de benzimidazóis nas diluições de 1:10 até 1:1280. $\mathrm{O} \mathrm{ABZ}^{1}$ e o OFZ revelaram pouca eficiência para as diluições de 1:40 até 1:5120, enquanto o FBZ não foi eficaz nas diferentes diluições usadas ( $P$ $>0,05)$. Entre os produtos testados, o IVM demonstrou uma melhor ação ovi-larvicida, observados principalmente entre as diluições 1:320 e 1:1280, porém os produtos LEV e CLS foram eficazes quanto ação ovi-larvicida na diluição 1:80.

Com relação ao gênero Haemonchus Tabela 2, não houve diferença na média do número de $\mathrm{L}_{3}$ entre os anti-helmínticos no grupo controle e diluições 1:1280 a 1:5120. Os produtos $\mathrm{ABZ}^{1}$, FBZ e OFZ não apresentaram diferenças significativas quanto ao número médio de $\mathrm{L}_{3}$ em nenhuma das diluições para $(\mathrm{P}>0,05)$. Enquanto que o $\mathrm{ABZ}^{2}$ revelou melhor ação letal para ovos de Haemonchus spp nas diluições de 1:10 a 1:80, dentre os quatro produtos a base de benzimidazóis utilizados. Os produtos IVM, LEV e CLS revelaram ação ovi-larvicida nas diluições de 1:10 a 1:640; 1:10 a 1:80 e 1:10 a 1:20, respectivamente.

Conforme Tabela 3, no gênero Trichostrongylus, a eficiência ovi-larvicida observada nas diluições utilizadas para os sete anti-helmínticos, foi semelhante à obtida para Haemonchus spp. Não houve diferença estatisticamente significativa no grupo controle e nas diluições 1:1280 a 1:5120 ( $\mathrm{P}>0,05)$, dos medicamentos usados. Contudo, o produto $\mathrm{ABZ}^{2}$ dentre os benzimidazóis mostrou a melhor eficiência ovi-larvicida até 1:80. O IVM apresentou uma eficácia ovi-larvicida total (100\%) para esse gênero até a diluição 1:640. Os produtos LEV e CLS quando comparados entre si, apresentaram ações ovi-larvicidas semelhantes nas diluições de 1:10 a 1:20.

$\mathrm{Na}$ Tabela 4, com relação ao gênero Oesophagostomum, todos os produtos apresentaram diferenças estatisticamente significativas $(\mathrm{P}<$ $0,05)$ quando comparadas ao grupo controle. Nas diluições 1:80 e no grupo controle não houve diferença na média do número de larvas entre os diferentes anti-helmínticos. A IVM apresentou ação ovi-larvicida até a diluição 1:640, o LEV e CLS se mostraram letais entre as diluições de 1:10 a 1:320 e 1:10 a 1:80, respectivamente.

\section{DISCUSSÃO}

O diagnóstico de resistência anti-helmíntica in vitro foi descrito en 1976 . Este método é rápido e aplicável na determinação de níveis de resistência, baseado na ação ovicida in vitro de anti-helmínticos benzimidazóis. Investigadores demonstraram, através do teste de eclosão in vitro, a resistência do $H$. contortus e $T$. colubriformis de ovinos a todos os benzimidazóis 
após seleção com thiabendazole ${ }^{8}$. Relatam que a resistência foi mais acentuada no teste in vitro do que in vivo. Concluiram que o teste de eclosão in vitro pode ser usado tanto para determinar a ocorrência como o nível relativo de resistência dos nematódeos aos anti-helmínticos benzimidazóis. Neste experimento verificou-se que a atividade in vitro de anti-helmínticos sobre larvas

Tabela 1. Médias do número de larvas infectantes $\left(L_{3}\right)$ de nematódeos gastrintestinais de caprinos obtidas por coprocultura quantitativa, tratadas com anti-helmínticos em diferentes diluições

\begin{tabular}{|c|c|c|c|c|c|c|c|c|}
\hline \multicolumn{9}{|c|}{ Benzimidazóis } \\
\hline \multirow{3}{*}{\multicolumn{2}{|c|}{$\begin{array}{l}\text { Anti-helmínticos } \\
\text { OPG / Controle } \\
\text { LDPG / Controle }\end{array}$}} & \multirow{4}{*}{$\begin{array}{r}\mathbf{A B Z} \mathbf{1}^{1} \\
9.550 \\
10.795^{\mathrm{cA}} \\
158,5^{\mathrm{aA}}\end{array}$} & \multirow{4}{*}{$\begin{array}{r}\text { FBZ } \\
7.200 \\
11.185^{\mathrm{aA}} \\
4.720,0^{\mathrm{aB}}\end{array}$} & \multirow{3}{*}{$\begin{array}{r}\text { OFZ } \\
7.650 \\
7.045^{\mathrm{dA}}\end{array}$} & \multirow{3}{*}{$\begin{array}{r}\mathrm{ABZ}^{2} \\
9.150 \\
9.235^{\mathrm{cA}}\end{array}$} & \multirow{3}{*}{$\begin{array}{r}\text { IVM } \\
7.500 \\
7.220^{\mathrm{bA}}\end{array}$} & \multirow{3}{*}{$\begin{array}{r}\text { LEV } \\
10.000 \\
7.200^{\mathrm{cA}}\end{array}$} & \multirow{3}{*}{$\begin{array}{r}\text { CLS } \\
9.250 \\
9.090^{\mathrm{cA}}\end{array}$} \\
\hline & & & & & & & & \\
\hline & & & & & & & & \\
\hline Média & $10 x$ & & & $128,0^{\mathrm{aA}}$ & $0^{\text {aA }}$ & $1,5^{\mathrm{aA}}$ & $2,5^{\mathrm{aA}}$ & $0^{\mathrm{aA}}$ \\
\hline do & $20 x$ & $463,5^{\mathrm{abA}}$ & $6.955,0^{\mathrm{aB}}$ & $411,0^{\mathrm{abA}}$ & $0^{\mathrm{aA}}$ & $7,5^{\mathrm{aA}}$ & $4,5^{\mathrm{aA}}$ & $0^{\mathrm{aA}}$ \\
\hline LDPG & $40 \mathrm{x}$ & $4.037,5^{\mathrm{bcA}}$ & $9.030,0^{\mathrm{aA}}$ & $625,0^{\text {abcAB }}$ & $0^{\mathrm{aB}}$ & $26,0^{\mathrm{aB}}$ & $158,5^{\mathrm{aB}}$ & $0^{\mathrm{aB}}$ \\
\hline por & $80 x$ & $9.485,0^{\mathrm{cA}}$ & $10.235,0^{\mathrm{aA}}$ & $1.605,0^{\mathrm{abcdAB}}$ & $14,5^{\mathrm{aC}}$ & $215,5^{\mathrm{aBC}}$ & 372,5 abBC & 569,5 abBC \\
\hline diluicao & $160 x$ & $9.905,0^{\mathrm{cA}}$ & $10.370,0^{\mathrm{aA}}$ & $3.607,5$ bcdAB & $457,5^{\mathrm{abB}}$ & $625,0^{\mathrm{aB}}$ & $3.716,0^{\text {bc } \mathrm{AB}}$ & $5.703,0^{\mathrm{bcA}}$ \\
\hline do & $320 x$ & $12.270,0^{\mathrm{cA}}$ & $11.355,0^{\mathrm{aA}}$ & $4.307,5^{\mathrm{bcdA}}$ & $3.047,5^{\mathrm{bcAB}}$ & $580,0^{\mathrm{aB}}$ & $4.007,5$ bcAB & $6.609,5 \mathrm{bcA}$ \\
\hline anti- & $640 x$ & $8.345,0^{\mathrm{cA}}$ & $10.030,0^{\mathrm{aA}}$ & $5.450,0^{\mathrm{cdA}}$ & $3.160,0^{\mathrm{bcA}}$ & $24,5^{\mathrm{aB}}$ & $3.342,5$ bcA & $10.640,0^{\mathrm{cA}}$ \\
\hline hel- & $1280 x$ & $10.230,0^{\mathrm{cA}}$ & $10.945,0^{\mathrm{aA}}$ & $6.530,0^{\mathrm{cdAB}}$ & $4.992,5^{\mathrm{bcAB}}$ & $602,5^{\mathrm{aB}}$ & $4.530,0^{\mathrm{bcAB}}$ & $9.815,0^{\mathrm{cA}}$ \\
\hline minti- & $2560 x$ & $10.650,0^{\mathrm{cA}}$ & $11.810,0^{\mathrm{aA}}$ & $6.505,0^{\mathrm{cdA}}$ & $6.430,0^{\mathrm{cA}}$ & $4.307,5^{\mathrm{bA}}$ & $6.515,0^{\mathrm{cA}}$ & $11.845,0^{\mathrm{cA}}$ \\
\hline co & $5120 x$ & $8.355,0^{\mathrm{cA}}$ & $9.390,0^{\mathrm{aA}}$ & $7.505,0 \mathrm{dA}$ & $8.905,0^{\mathrm{cA}}$ & $7.973,5^{\mathrm{bA}}$ & $8.002,0^{\mathrm{cA}}$ & $7.385,0^{\mathrm{cA}}$ \\
\hline
\end{tabular}

$\mathrm{ABZ}^{1}=$ Albendazole, $\mathrm{FBZ}=$ Fenbendazole, $\mathrm{OFZ}=$ Oxfendazole, $\mathrm{ABZ}^{2}=$ Sulfoxide de Albendazole, $\mathrm{IVM}=\mathrm{Ivermectin}$, LEV = Levamisole, CLS = Closantel.

Letras minúsculas diferentes nas colunas das médias indicam diferenças significativas $(\mathrm{P}<0,05)$.

Letras maiúsculas diferentes nas linhas das médias indicam diferenças significativas $(\mathrm{P}<0,05)$.

Média de OPG = Ovos por Grama de Fezes.

Média de LDPG = Larvas Desenvolvidas por Grama de Fezes / Testemunha.

Tabela 2. Médias do número de larvas infectantes $\left(\mathrm{L}_{3}\right)$ do gênero Haemonchus obtida por coprocultura quantitativa, tratados com anti-helmínticos em diferentes diluições.

\begin{tabular}{|c|c|c|c|c|c|c|c|c|}
\hline & & & & Benzimida & & & & \\
\hline Anti-helm & & $\mathbf{A B Z}^{1}$ & FBZ & OFZ & $\mathbf{A B Z} Z^{2}$ & IVM & LEV & CLS \\
\hline OPG/Cont & & 9.550 & 7.200 & 7.650 & 9.150 & 7.500 & 10.000 & 9.250 \\
\hline LDPG/Con & & $10.795^{\mathrm{aA}}$ & $11.185^{\mathrm{aA}}$ & $7.045^{\mathrm{aA}}$ & $9.235^{\mathrm{bA}}$ & $7.220 \mathrm{bA}$ & $7.200^{\mathrm{bA}}$ & $9.090 \mathrm{cA}$ \\
\hline Média do & $10 x$ & $8.308,0^{\mathrm{aA}}$ & $6.451,0^{\mathrm{aA}}$ & $4.233,0^{\mathrm{aA}}$ & $0^{\mathrm{aB}}$ & $0^{\mathrm{aB}}$ & $0^{\mathrm{aB}}$ & $0^{a B}$ \\
\hline LDPG por & $20 x$ & $7.752,0^{\mathrm{aA}}$ & $7.733,5^{\mathrm{aA}}$ & $4.977,5^{\mathrm{aA}}$ & $0^{\mathrm{aB}}$ & $0^{\mathrm{aB}}$ & $0^{\mathrm{aB}} 0^{\mathrm{aB}}$ & \\
\hline diluicao & $40 x$ & $7.524,5^{\mathrm{aA}}$ & $7.096,5^{\mathrm{aA}}$ & $5.006,0^{\mathrm{aA}}$ & $0^{\mathrm{aB}}$ & $0^{\mathrm{aB}}$ & $0^{\mathrm{aB}}$ & $4.240,5^{\mathrm{bA}}$ \\
\hline & $80 x$ & $7.423,5^{\mathrm{aA}}$ & $7.795,5^{\mathrm{aA}}$ & $3.322,0 \mathrm{aA}$ & $0^{\mathrm{aB}}$ & $0^{\mathrm{aB}}$ & $0^{\mathrm{aB}}$ & $7.786,0^{\mathrm{cA}}$ \\
\hline & $160 x$ & $7.700,5^{\mathrm{aA}}$ & $7.385,5^{\mathrm{aA}}$ & $5.152,0^{\mathrm{aA}}$ & $6.157,0^{\mathrm{bA}}$ & $0^{\mathrm{aB}}$ & $1.054,5 \mathrm{aB}$ & $4.953,5 \mathrm{bcA}$ \\
\hline & $320 x$ & $7.545,0^{\mathrm{aA}}$ & $6.506,0^{\mathrm{aA}}$ & $4.964,0$ aA & $5.871,0^{\mathrm{bA}}$ & $0^{\mathrm{aB}}$ & $2.975,0^{\mathrm{bA}}$ & $4.971,0^{\mathrm{bcA}}$ \\
\hline & $640 x$ & $8.291,0^{\mathrm{aA}}$ & $6.494,5^{\mathrm{aA}}$ & $3.455,0^{\mathrm{aA}}$ & $5.721,5^{\mathrm{bA}}$ & $0^{\mathrm{aB}}$ & $4.138,5^{\mathrm{bA}}$ & $5.236,5 \mathrm{bcA}$ \\
\hline & $1280 x$ & $7.669,0^{\mathrm{aA}}$ & $6.214,0^{\mathrm{aA}}$ & $4.308,0$ aA & $6.010,5^{\mathrm{bA}}$ & $4.648,0^{\mathrm{bA}}$ & $4.395,0^{\mathrm{bA}}$ & $5.308,0^{\mathrm{bcA}}$ \\
\hline & $2560 x$ & $7.268,5^{\mathrm{aA}}$ & $5.889,5^{\mathrm{aA}}$ & $3.747,5^{\mathrm{aA}}$ & $5.012,5^{\mathrm{bA}}$ & $4.633,0^{\mathrm{bA}}$ & $4.093,5^{\mathrm{bA}}$ & $4.254,5^{\mathrm{bcA}}$ \\
\hline & $5120 x$ & $6.744,5^{\mathrm{aA}}$ & $5.945,5^{\mathrm{aA}}$ & $3.747,5^{\mathrm{aA}}$ & $4.745,0^{\mathrm{bA}}$ & $4.292,0^{\mathrm{bA}}$ & $3.860,5^{\mathrm{bA}}$ & $5.352,5 \mathrm{bcA}$ \\
\hline
\end{tabular}

$\mathrm{ABZ}^{1}=$ Albendazole, $\mathrm{FBZ}=$ Fenbendazole, $\mathrm{OFZ}=$ Oxfendazole, $\mathrm{ABZ}^{2}=$ Sulfoxide de Albendazole, $\mathrm{IVM}=\mathrm{Ivermectin}$, $\mathrm{LEV}=$ Levamisole, $\mathrm{CLS}=$ Closantel.

Letras minúsculas diferentes nas colunas das médias indicam diferenças significativas $(\mathrm{P}<0,05)$.

Letras maiúsculas diferentes nas linhas das médias indicam diferenças significativas $(\mathrm{P}<0,05)$.

Média de OPG = Ovos por Grama de Fezes.

Média de LDPG = Larvas Desenvolvidas por Grama de Fezes / Testemunha. 
Tabela 3. Médias do número de larvas infectantes $\left(L_{3}\right)$ do gênero Trichostrongylus obtida por coprocultura quantitativa, tratados com anti-helmínticos em diferentes diluições

\begin{tabular}{|c|c|c|c|c|c|c|c|c|}
\hline \multicolumn{9}{|c|}{ Benzimidazóis } \\
\hline \multirow{3}{*}{\multicolumn{2}{|c|}{$\begin{array}{l}\text { Anti-helmínticos } \\
\text { OPG/Controle } \\
\text { LDPG/Controle }\end{array}$}} & $\mathbf{A B Z}^{1}$ & FBZ & OFZ & $\mathrm{ABZ}^{2}$ & IVM & LEV & CLS \\
\hline & & 9.550 & 7.200 & 7.650 & 9.150 & 7.500 & 10.000 & 9.250 \\
\hline & & $10.795^{\mathrm{aA}}$ & $11.185^{\mathrm{aA}}$ & $7.045^{\mathrm{aA}}$ & $9.235^{\mathrm{bA}}$ & $7.220^{\mathrm{bA}}$ & $7.200^{\mathrm{bA}}$ & $9.090^{\mathrm{cA}}$ \\
\hline Média do & $10 x$ & $2.654,5^{\mathrm{aA}}$ & $4.733,5^{\mathrm{aA}}$ & $2.812,0^{\mathrm{aA}}$ & $0^{\mathrm{aB}}$ & $0^{\mathrm{aB}}$ & $0^{\text {aB }}$ & $0^{\mathrm{aB}}$ \\
\hline LDPG & $20 x$ & $3.043,0$ aA & $3.476,5^{\mathrm{aA}}$ & $2.067,5^{\mathrm{aA}}$ & $0^{\mathrm{aB}}$ & $0^{\mathrm{aB}}$ & $0^{\mathrm{aB}}$ & $0^{\mathrm{aB}}$ \\
\hline por & $40 x$ & $3.214,5^{\mathrm{aA}}$ & $4.088,0^{\mathrm{aA}}$ & $2.040,0^{\mathrm{aA}}$ & $0^{\mathrm{aB}}$ & $0^{\mathrm{aB}}$ & 0 aв & $400,0^{\mathrm{aAB}}$ \\
\hline \multirow[t]{7}{*}{ diluicia } & $80 x$ & $3.371,0$ aA & $3.389,5^{\mathrm{aA}}$ & $1.730,0^{\mathrm{aA}}$ & $0^{\mathrm{aB}}$ & $0^{\mathrm{aB}}$ & $3.515,0^{\mathrm{bA}}$ & $1.303,5^{\mathrm{bA}}$ \\
\hline & $160 x$ & $3.094,5^{\mathrm{aA}}$ & $3.799,5^{\mathrm{aA}}$ & $1.893,0^{\mathrm{aA}}$ & $3.054,5^{\mathrm{bA}}$ & $0^{\mathrm{aB}}$ & $3.409,5^{\mathrm{bA}}$ & $2.685,5^{\mathrm{bA}}$ \\
\hline & $320 x$ & $3.249,5$ aA & $3.956,5^{\mathrm{aA}}$ & $2.081,0^{\mathrm{aA}}$ & $3.821,5^{\mathrm{bA}}$ & $0^{\mathrm{aB}}$ & $3.832,5^{\mathrm{bA}}$ & $2.593,5^{\mathrm{bA}}$ \\
\hline & $640 x$ & $5.066,0^{\mathrm{aA}}$ & $7.753,5^{\mathrm{aA}}$ & $3.455,0^{\mathrm{aA}}$ & $3.513,5^{\mathrm{bA}}$ & $0^{\mathrm{aB}}$ & $2.386,5^{\mathrm{bA}}$ & $2.072,5^{\mathrm{bA}}$ \\
\hline & $1280 \mathrm{x}$ & $7.669,0$ aA & $7.161,5^{\mathrm{aA}}$ & $4.308,0$ aA & $3.224,5 \mathrm{bA}$ & $2.423,0^{\mathrm{bA}}$ & $1.961,5 \mathrm{bA}$ & $2.341,0^{\mathrm{bA}}$ \\
\hline & $2560 x$ & $7.268,5^{\mathrm{aA}}$ & $7.749,5^{\mathrm{aA}}$ & $3.747,5^{\mathrm{aA}}$ & $4.222,0^{\mathrm{bA}}$ & $1.714,5^{\mathrm{bA}}$ & $2.464,5^{\mathrm{bA}}$ & $3.443,0^{\mathrm{bA}}$ \\
\hline & $5120 x$ & $6.744,5^{\mathrm{aA}}$ & $7.157,0^{\mathrm{aA}}$ & $4.050,5^{\mathrm{aA}}$ & $4.489,5^{\mathrm{bA}}$ & $1.484,5^{\mathrm{bA}}$ & $2.475,0^{\mathrm{bA}}$ & $2.720,0^{\mathrm{bA}}$ \\
\hline
\end{tabular}

$\mathrm{ABZ}^{1}=$ Albendazole, $\mathrm{FBZ}=$ Fenbendazole, $\mathrm{OFZ}=$ Oxfendazole, $\mathrm{ABZ}^{2}=$ Sulfoxide de Albendazole, $\mathrm{IVM}=\mathrm{Ivermectin}$, $\mathrm{LEV}=$ Levamisole, $\mathrm{CLS}=$ Closantel.

Letras minúsculas diferentes nas colunas das médias indicam diferenças significativas $(\mathrm{P}<0,05)$.

Letras maiúsculas diferentes nas linhas das médias indicam diferenças significativas $(\mathrm{P}<0,05)$.

Média de OPG = Ovos por Grama de Fezes.

Média de LDPG = Larvas Desenvolvidas por Grama de Fezes / Testemunha.

Tabela 4. Médias do número de larvas infectantes $\left(\mathrm{L}_{3}\right)$ do gênero Oesophagostomum obtida por coprocultura quantitativa, tratados com anti-helmínticos em diferentes diluições

\begin{tabular}{|c|c|c|c|c|c|c|c|c|}
\hline & & & & & zzimidaz & & & \\
\hline Anti-hel & nínticos & $\mathbf{A B Z} \mathbf{Z}^{1}$ & FBZ & OFZ & $\mathbf{A B Z} Z^{2}$ & IVM & LEV & CLS \\
\hline OPG / C & ontrole & 9.550 & 7.200 & 7.650 & 9.150 & 7.500 & 10.000 & 9.250 \\
\hline LDPG / & Controle1 & $0.795^{\mathrm{bA}}$ & $11.185^{b A}$ & $7.045^{b A}$ & $9.235^{\mathrm{bA}}$ & $7.220 \mathrm{cA}$ & $7.200 \mathrm{cA}$ & $9.0900^{\mathrm{cA}}$ \\
\hline Média do & $10 x$ & $0^{\text {aA }}$ & $0^{\mathrm{aA}}$ & $0^{\mathrm{aA}}$ & $0^{\text {aA }}$ & $0^{\text {aA }}$ & $0^{\mathrm{aA}}$ & $0^{\text {aA }}$ \\
\hline LDPG & $20 x$ & $0^{\mathrm{aA}}$ & $0^{\text {aA }}$ & $0^{\text {aA }}$ & $0^{\text {aA }}$ & $0^{\text {aA }}$ & $0^{\mathrm{aA}}$ & $0^{\text {aA }}$ \\
\hline por & $40 x$ & $0^{\mathrm{aA}}$ & $0^{\mathrm{aA}}$ & $0^{\mathrm{aA}}$ & $0^{\mathrm{aA}}$ & $0^{\text {aA }}$ & $0^{\mathrm{aA}}$ & 179,0 abA \\
\hline diluicao & $80 x$ & $0^{\text {aA }}$ & $0^{\mathrm{aA}}$ & $0^{\mathrm{aA}}$ & $0^{\text {aA }}$ & $0^{\text {aA }}$ & $0^{\mathrm{aA}}$ & $0^{\text {aA }}$ \\
\hline & $160 x$ & $0^{\mathrm{aA}}$ & $0^{\text {aA }}$ & $0^{\text {aA }}$ & $0^{\text {aA }}$ & $0^{\text {aA }}$ & $0^{\mathrm{aA}}$ & $1.445,5 \mathrm{bcB}$ \\
\hline & $320 x$ & $0^{\mathrm{aA}}$ & $0^{\mathrm{aA}}$ & $0^{\mathrm{aA}}$ & $0^{\mathrm{aA}}$ & $0^{\text {aA }}$ & $0^{\mathrm{aA}}$ & $1.525,0^{\mathrm{bcB}}$ \\
\hline & $640 x$ & $0^{\text {aA }}$ & $0^{\mathrm{aA}}$ & $0^{\mathrm{aA}}$ & $0^{\mathrm{aA}}$ & $0^{\text {aA }}$ & $396,5^{\mathrm{abAB}}$ & $1.465,0 \mathrm{bcB}$ \\
\hline & $1280 x$ & $0^{\mathrm{aA}}$ & $0^{\mathrm{aA}}$ & $0^{\mathrm{aA}}$ & $224,0^{\mathrm{aA}}$ & 747,0 abA & 394,5 abA & $1.003,5 \mathrm{abA}$ \\
\hline & $2560 x$ & $0^{\text {aA }}$ & $0^{\mathrm{aA}}$ & $0^{\mathrm{aA}}$ & $0^{\mathrm{aA}}$ & 872,0 bcB & 715,0 abcAB & $1.392,5^{\mathrm{bcB}}$ \\
\hline & $5120 x$ & $0^{\text {aA }}$ & $179,5^{\mathrm{aAB}}$ & $0^{\mathrm{aA}}$ & $0^{\text {aA }}$ & $1.321,0 \mathrm{bcB}$ & 864,0 abcAB & $1.017,0 \mathrm{abAB}$ \\
\hline
\end{tabular}

$\mathrm{ABZ}^{1}=$ Albendazole, $\mathrm{FBZ}=$ Fenbendazole, $\mathrm{OFZ}=$ Oxfendazole, $\mathrm{ABZ}^{2}=$ Sulfoxide de Albendazole, $\mathrm{IVM}=\mathrm{Ivermectin}$, LEV = Levamisole, CLS $=$ Closantel.

Letras minúsculas diferentes nas colunas das médias indicam diferenças significativas $(\mathrm{P}<0,05)$.

Letras maiúsculas diferentes nas linhas das médias indicam diferenças significativas $(\mathrm{P}<0,05)$.

Média de OPG = Ovos por Grama de Fezes.

Média de LDPG = Larvas Desenvolvidas por Grama de Fezes / Testemunha.

infectantes $\left(\mathrm{L}_{3}\right)$ de nematódeos gastrintestinais de caprinos, utilizando-se a técnica de coprocultura quantitativa ${ }^{5}$ foi eficiente, pois demonstrou através dos resultados obtidos qual dos anti-helmínticos foi eficaz.
Infelizmente, não existe, no Brasil, uma legislação adequada para o registro de antihelmínticos, sendo frequentemente registradas e comercializadas novas drogas com subdoses e sem teste de avaliação no país ${ }^{9,10}$. Para maior 
segurança, com relação à eficácia dos antihelmínticos utilizados, seria prudente que, antes do aceite dos resultados da avaliação do princípio ativo, outros testes fossem realizados, utilizandose um número significativo de animais experimentais, em várias localidades e com várias estirpes de nematódeos ${ }^{11}$.

\section{RESUMO}

Atividade in vitro de anti-helmínticos sobre larvas infectantes $\left(\mathrm{L}_{3}\right)$ de nematódeos gastrintestinais de caprinos, utilizando a Técnica de Coprocultura Quantitativa. Este trabalho foi realizado com o objetivo de comparar a ação ovilarvicida in vitro de anti-helmínticos, através da técnica de coprocultura quantitativa para nematódeos gastrintestinais de caprinos. Foram testados sete anti-helmínticos no experimento: albendazole, sulfoxido de albendazole, fenbendazole, oxfendazole, ivermectin, levamisole e closantel. Os caprinos utilizados para obtenção de fezes, foram mantidos na Escola de Medicina Veterinária/Universidade Federal da Bahia(UFBA), por um ano e meio, sem dosificações antihelmínticas. Os anti-helmínticos foram diluídos em água destilada, a partir da solução original, iniciando-se da diluição 1:10 até 1:5120, perfazendo no total de 10 diluições.

As coproculturas preparadas com $2 \mathrm{~g}$ de fezes, $2 \mathrm{~g}$ de serragens e $2 \mathrm{ml}$ de água destilada ou das diferentes diluições dos anti-helmínticos foram mantidos a $34^{\circ} \mathrm{C}$, por sete dias, para obtenção das larvas infectantes de nematóides gastrintestinais. Foram realizadas as comparações dos números de $\mathrm{L}_{3}$ de Strongyloidea por gênero e diluições de anti-helmínticos entre os grupos de testes e controle. A Ivermectin apresentou melhor ação ovi-larvicida, com redução de larvas dos gêneros Haemonchus, Trichostrongylus e Oesophagostomum $\left(\mathrm{L}_{3}\right)$, enquanto o fenbendazole foi o produto com menor ação para os gêneros Haemonchus e Trichostrongylus. Para o gênero
Oesophagostomum, os produtos albendazole, fenbendazole, oxfendazole e sulfoxido de albendazole, eliminaram completamente a população dessa espécie. Entre os produtos ivermectin, levamisole e closantel, o ivermectin apresentou melhor ação ovi-larvicida para ovos de Oesophagostomum

\section{REFERÊNCIAS}

1.- IBGE - Instituto brasileiro de Geografia e Estatística. Produção da pecuária Municipal - Rio de Janeiro, 1995.

2.- UENO H, ARAUJO F R, BORGES C C L, D'ÁLMEIDA V A. Coprocultura_quantitativa para larvas de Strongyloidea em nematódeos gastrintestinais de caprinos. [Salvador]. Universidade Federal da Bahia. Escola de Medicina Veterinária, 1997. p. 19.

3.- GORDON $\mathrm{H} \mathrm{Mcl}$, WHITLOCK H V. A new tecnique for counting nematode eggs in sheep faeces. J Counc Sci Ind Res 1939; 12: 50-2.

4.- WHITLOCK H V. Some modifications of the McMaster eggs counting technique and apparatus. J Counc Sci Ind Res 1948; 21; 177-80.

5.- UENO H. Cultivo quantitativo de larvas de nematódeos gastrintestinais de ruminantes com tentativa para prédiagnóstico. Tokyo, Japan, 1995. p. 138.

6.- DICKMANS G, ANDREWS J S A. Comparative morphological study on the injective larvae of the common parasitic nematodes in the alimentary tract of sheep. Amer Micro Soc 1933; 52: 1-25.

7.- LE JAMBRE L F. Egg hatch as na in vitro assay of thiabendazole resistence in nematodes. Vet Parasitol 1976; 2: 385-91.

8.- HALLC A, CAMPBELL N J, RICHARDSON N J. Levels of benzimidazole resistance in Haemonchus contortus and Trichostrongylus colubriformis recorded from an egg hatch test procedure. Res Vet Sci 1978; 25: 360-3.

9.- PINHEIRO A C. Verminose ovina. In : Curso sobre parasitodos rumientes, 1, Lages-SC, 1981. Anais... Florianópolis-SC : Colégio Brasileiro de Parasitologia Veterinária, 1981; p. 61-75.

10.- HONER M R, BIANCHIN I. Considerações básicas para um programa de controle estratégico da verminose bovina em gado de corte no Brasil. Campo Grande: EMBRAPA- CNPGC. 53p. 1987. (EMBRAPACNPGC. Circular técnica, 20).

11.- BIANCHIN I. Alguns fatores que interferem no controle de helmintos de bovinos. In: Seminario Nacional sobre parasitoses em bovinos 1, Campo Grande-MS, Anais...Campo Grande: EMBRAPA - CNPQ,1979. p. 99-111. 